\title{
Development of quality functionals of kinetic processes for the synthesis of composite materials with specified parameters of structure and properties
}

\author{
$A$ Bormotov ${ }^{1, *}$ \\ ${ }^{1}$ Penza State Technological University, Penza, Russia
}

\begin{abstract}
The processes of structure formation and features of polymer composites with special properties are investigated, the main kinetic processes that have a decisive influence on the structure formation and properties of polymer composites are identified. The expediency of considering kinetic (transient) processes as a necessary and sufficient sign of the structure formation of a thermosetting is substantiated. A mathematical method for the synthesis of polymer composite materials of increased density using the quality functionals of kinetic (transient) processes is proposed. The proposed mathematical methods and models allow to effectively solve the problem of plasticization and modification of high-viscosity polymers, as well as to carry out multi-criteria synthesis of composite materials with strictly specified parameters of structure and properties using the quality functionals of kinetic (transient) processes.
\end{abstract}

\section{Introduction}

In recent years, polymer concretes and polymer mortars have been widely introduced into construction practice. They are used for corrosion protection of building structures (mastics, putties, adhesives, polymer concrete, polymer mortars).Polyester polymer concretes are of considerable interest. This is due to the fact that unsaturated polyesters are cured not only at elevated but also at room temperatures without the release of any by-products. Unsaturated polyester resins, due to their low initial viscosity, are easily processed at low pressures and temperatures. Polyesters can be combined with fillers of various basicities. With the help of pigments, you can give any color to the finished product [1].

Polymer concretes and polymer solutions based on unsaturated polyester resins are used for the installation of monolithic self-leveling floors and all kinds of protective coatings. The domestic industry produces a number of unsaturated polyester resins. However, most polyester resins of domestic and foreign brands cannot be used without modification for installing monolithic floors and protecting technological containers from aggressive influences. This is due to their high shrinkage with a significant modulus of elasticity, which leads to cracking of thin coatings based on such resins and to peeling of thick ones. The

\footnotetext{
* Corresponding author: aleks21618@yandex.ru
} 
widespread use of polyester resins in construction is hampered by the insufficient water and alkali resistance of compositions based on them [1].

\section{Materials and methods}

The creation of polyester quartz-containing composites, devoid of these disadvantages, is an extremely urgent task. One of the ways to solve it is the modification of fillers or binder with surface-active agents (SAA).

The basic provisions of the polystructural theory are used for developing polymer composite materials [2].

All composite building materials are considered as two-component systems, built on the principle of "structure in structure". The performance properties of composites are determined by the features of the micro- and macrostructure and their quantitative ratios. The properties of the microstructure are determined by the surface interaction forces in the polymer-filler contact and depend on the molecular, capillary and electrostatic forces. For example, an increase in the dispersion of a filler leads to an increase in its activity. Intermolecular interaction is determined by the chemical nature of the binder and filler, the nature of the bonds in contact.

According to the polystructural theory of composite building materials, the durability of a microstructure is determined by the volumetric concentration, specific surface area and activity of the filler, as well as porosity. SAA change the free surface energy of the components of the microstructure, affect the nature of the supramolecular structures [2].

\section{Main results}

The most promising for solving this problem is the use of dynamic models defined in the class of differential equations. For homogeneous systems, such a model in the class of ordinary differential equations is partially considered in [3-6]. Increasing the order of the differential equation makes it possible to describe kinetic processes in heterogeneous, dispersed, and polydisperse systems for many controlled parameters. In this case, as a rule, it turns out to be possible to restrict oneself to the class of ordinary differential equations with constant coefficients. In particular, for processes of the form shown in Figure 1, with the asymptotic output of the controlled parameter to the operational value, a model of the form [7] can be used:

$$
\ddot{x}+2 n \dot{x}+\omega_{o}^{2}\left(x-x_{m}\right)=0
$$

Here, the abscissa of the inflection point is defined as

$$
t_{n}=\frac{1}{\lambda_{1}-\lambda_{2}} \ln \frac{\lambda_{1}}{\lambda_{2}}
$$

here $\lambda_{1}, \lambda_{2}\left(\lambda_{1}>\lambda_{2}\right)$ - modulus of the roots of the (negative) characteristic polynomial

The value $\lambda_{2}$ can be determined by the end of the transient.

Having solved the equation connecting $r=\frac{\lambda_{1}}{\lambda_{2}}$ with $v=\lambda_{2} t_{n}$, which has the form: 


$$
r^{\frac{1}{r-1}}=e^{v}
$$

it is possible to determine the value $\lambda_{I}$, and therefore, taking into account the previous one, the process $x(t)$.

It is easy to determine experimentally the dependences $\lambda_{1}, \lambda_{2}, x_{m}$ (the process $\left.x(t)\right)$ on the model parameters $\omega_{o}$ and $n$ (instead of $n$, a dimensionless damping coefficient can be used $\xi=\frac{n}{\omega_{o}} \geq 1$ ).

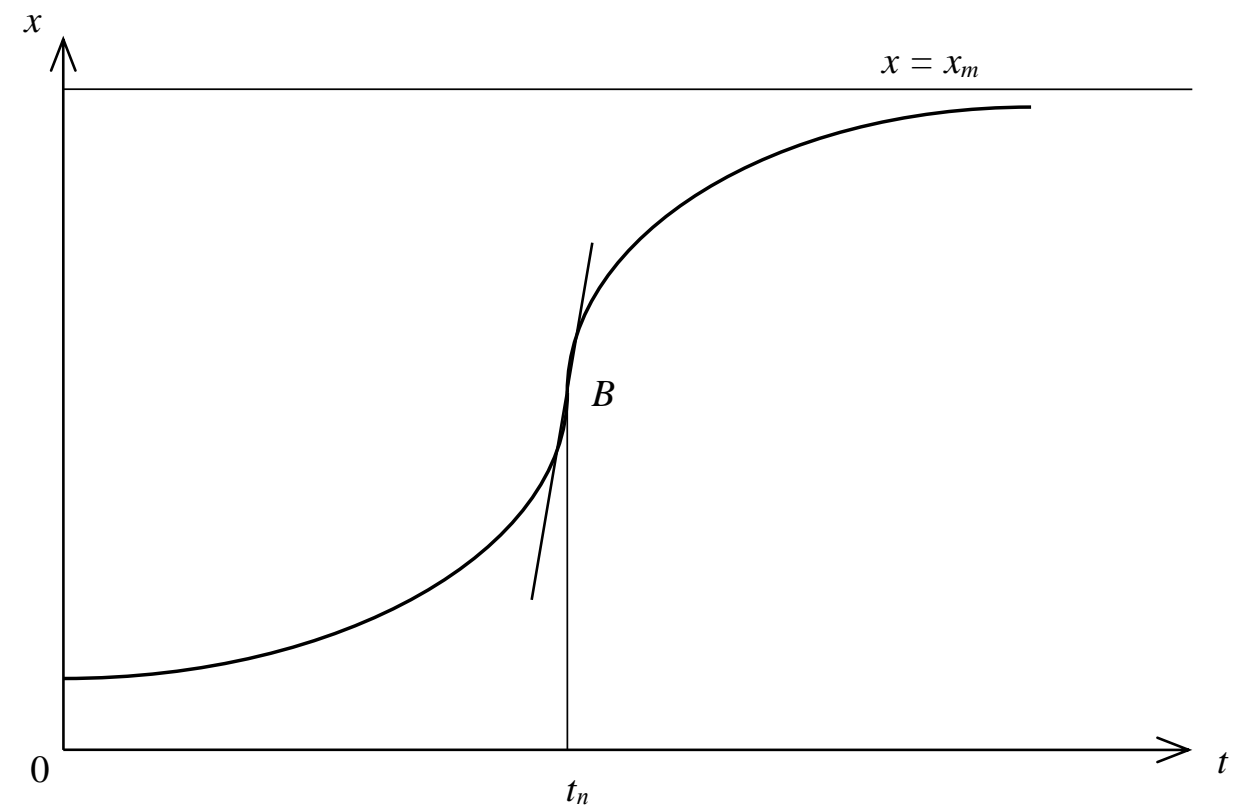

Fig. 1. Graph of transients.

The nature of the structure formation process is determined by the roots of the characteristic polynomial. Therefore, it seems natural to use the quality functional of the form as an additive global criterion in the synthesis of polymer composites:

$$
\Phi(S)=f \min _{i}\left\{\lambda_{i}\right\}+a \frac{1}{\min _{i}\left\{\lambda_{i}\right\}}+b \frac{\max _{i}\left\{\lambda_{i}\right\}}{\min _{i}\left\{\lambda_{i}\right\}}+c \frac{\min _{i}\left\{\lambda_{i}\right\}}{\max _{i}\left\{\lambda_{i}\right\}}
$$

In particular, for a second-order model:

$$
\Phi(S)=\left(\xi-\sqrt{\xi^{2}-1}\right) \omega_{o}+\frac{a}{\left(\xi-\sqrt{\xi^{2}-1}\right) \omega_{o}}+\frac{b\left(\xi+\sqrt{\xi^{2}-1}\right)}{\left(\xi-\sqrt{\xi^{2}-1}\right)}+\frac{c\left(\xi-\sqrt{\xi^{2}-1}\right)}{\left(\xi+\sqrt{\xi^{2}-1}\right)} .
$$

The proposed quality functional allows the synthesis of polymer composite materials. To do 
this, you need to build level lines $\Phi(S)=$ const, which are the boundaries of areas of equal material estimates. Then, to improve the quality of the material, the structure and properties of the components should be changed in accordance with the parameters of the model, moving in an anti-gradient direction perpendicular to the level line.

In principle, the weight constants in the quality functional can be determined by the method of expert assessments, however, it is more expedient taking into account the correlations between $\lambda_{i}$ and

$\Phi(S)$.

\section{Considerations}

A monomolecular surfactant layer around the filler grain improves the wetting of the filler with a polyester resin, which increases the adhesion of the binder to the filler surface, changes the nature of the pores, and reduces the volume of air inclusions at the polymer-filler interface. Under the influence of surfactants, relaxation processes change, resulting in a decrease in internal shrinkage and temperature stresses. The kinetics of structure formation also changes, positively affecting the nature of supramolecular structures. The sizes of the supramolecular formations decrease.

Globular structures of polyester composites transform into fibrillar structures, causing increased extensibility at high values of adhesion of the binder to the filler surface [2].

The adsorbed surfactant layer improves the polymer wetting the filler surface, increases the adhesion of the binder to the filler surface, reduces internal stresses, since it plays the role of an elastic layer between the solid filler surface and polymer macromolecules, improves the conditions for relaxation processes. To increase the water resistance, polyester composites are transferred to the electret state. Electrothermal and magnetoelectrets with improved structure have been developed.

The macrostructure properties of the composite are determined by the packing density of the filler grains. The role of surface forces is less significant here. Compaction of the macrostructure reaches its maximum when the grains of the fine fraction are located in the voids of the coarse fraction without the expansion of large grains [8]. The density of the macrostructure can be characterized by the packing factor. The macro-structure of the composite is optimal if the aggregate grains are arranged uniformly throughout the volume and with a minimum spreading ratio. In this case, it is assumed that the microstructure has the form of a continuous interlayer at the optimal $\mathrm{P} / \mathrm{N}$ ratio, ensuring the presence of the binder in a thin film-like state. The structure-forming factors of the macrostructure are the thickness of the contact layer of the microstructure, the coefficient of separation of grains, and the $\mathrm{P} / \mathrm{N}$ ratio $[8,9]$. The optimal structure of the composite corresponds to the increased density of the material, as a rule, with increased strength indicators. Optimization of the structure and properties of composite materials based on the polystructural theory is reduced to the optimization of the micro- and macrostructure. On the basis of the well-known law of alignment, optimization can be performed according to the corresponding indicator [1-2, 89].

As we see, optimization of the structure and properties of composite materials is largely associated with the construction of models of kinetic processes that take into account the change in the controlled parameters at different stages of structure formation with the simultaneous determination of the estimate of the kinetic parameters corresponding to the desired process. These tasks include determining the effect of the type and dispersion of the filler on the processes of structure formation and properties of composites, modification of the binder or the surfactant composite itself in order to increase, for example, the resistance to aggressive media that affect the curing processes, reduce shrinkage and internal stresses, improve physical, mechanical and operational properties [10-11]. Models of kinetic 
processes, built on the basis of fractal geometry, using exponential relationships, including modified exponents, Pearl's logistic curve [11], are known.

Basically, these models play only the role of interpolation and are not used enough to create new building materials. The possibility of using these models is very limited. For the approximation of kinetic processes in heterogeneous systems, the logistic curve is often used

$$
x\left(t, \alpha_{1}, \alpha_{2}, \alpha_{3}\right)=\frac{\alpha_{1}}{1+\exp \left(\alpha_{2}-\alpha_{3} t\right)}, t=1,2, \ldots, n \geq 3
$$

There:

$$
\dot{x}(t)=\frac{\alpha_{1} \alpha_{3} u}{(1+u)^{2}}, \quad \ddot{x}(t)=\frac{\alpha_{1} \alpha_{3}^{2} u(u-1)}{(1+u)^{3}}, \quad \dddot{x}(t)=\frac{\alpha_{1} \alpha_{3}^{3} u}{(1+u)^{4}}\left(u^{2}-4 u+1\right)
$$

here $u=e^{\left(\alpha_{2}-\alpha_{3} t\right)}$

Whence the abscissa of point B (Figure 2) - the inflection point - is found from the condition $\ddot{x}\left(t_{B}\right)=0$, that gives $t_{B}=\frac{\alpha_{2}}{\alpha_{3}}$.

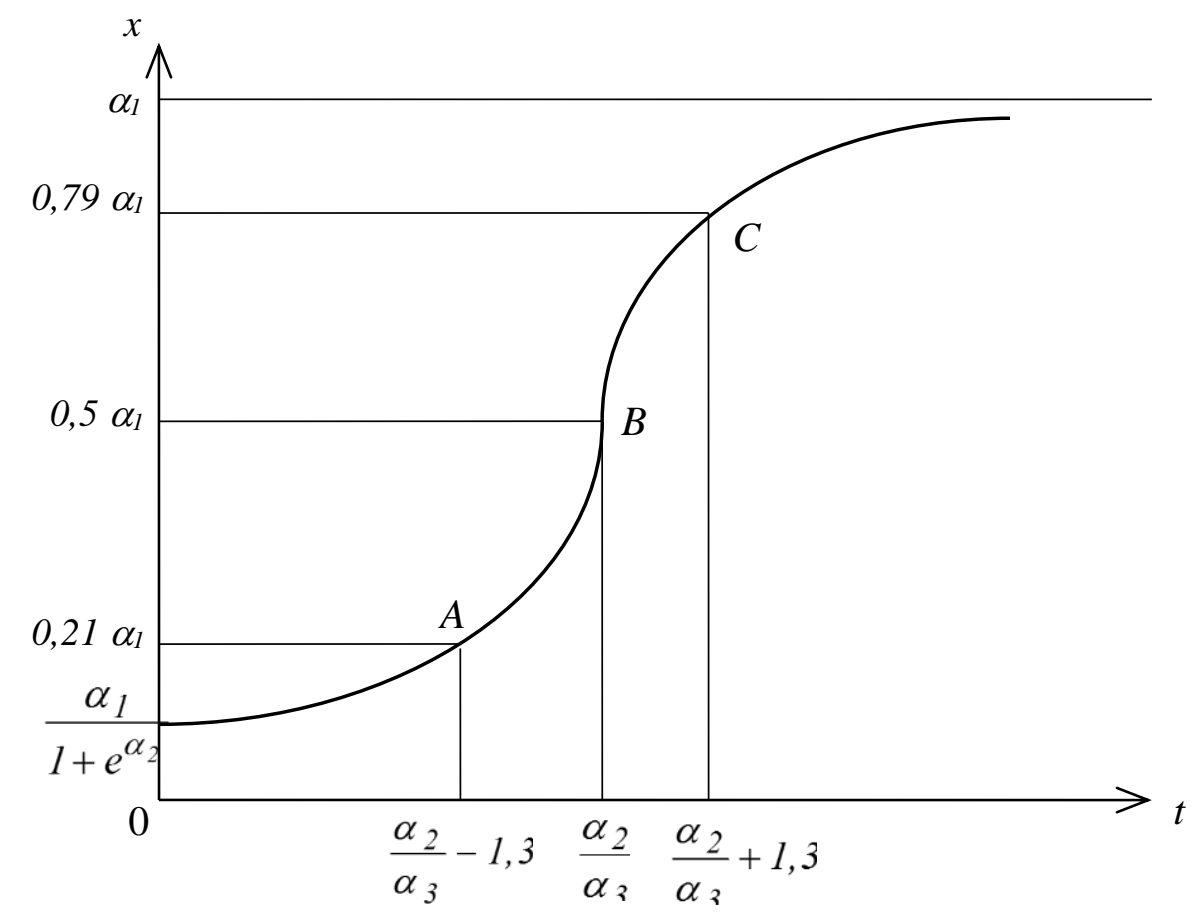

Fig. 2. Logistic curve.

Moreover, the value $x(t)$ at point $B x\left(t_{B}\right)=\frac{\alpha_{1}}{2}$, and does not depend on $\alpha_{2}$ and $\alpha_{3}$. For any $\alpha_{3} x(0)=\frac{\alpha_{1}}{1+e^{\alpha_{2}}}$.

From the above it follows the limited possibility of using the logistic curve to approximate 
the kinetic processes of structure formation of composites, since in many cases $x(0) \approx 0$, $x\left(t_{B}\right) \neq \frac{\alpha_{1}}{2}=\frac{x_{m}}{2}$.

This is also confirmed by the following. The abscissas of points $A, C$ are determined from the condition $\dddot{x}=0$, that gives

$$
u^{2}-4 u+1=0
$$

Whence

$$
u_{1,2}=2 \pm \sqrt{3}
$$

Must have:

$$
\begin{gathered}
e^{\alpha_{2}-\alpha_{3} t}=2 \pm \sqrt{3} \\
\alpha_{2}-\alpha_{3} t=\ln (2 \pm \sqrt{3})
\end{gathered}
$$

Whence

$$
t_{A}=\frac{\alpha_{2}-\ln (2+\sqrt{3})}{\alpha_{3}}=\frac{\alpha_{2}}{\alpha_{3}}-\frac{\ln (2+\sqrt{3})}{\alpha_{3}} \approx t_{B}-\frac{1,32}{\alpha_{3}} .
$$

Analogically

$$
t_{C} \approx t_{B}+\frac{1,32}{\alpha_{3}}
$$

As you can see, points $A$ and $C$ are symmetric about $B$. Wherein

$$
\begin{gathered}
x\left(t_{A}\right)=\frac{\alpha_{1}}{1+e^{\alpha_{2}-\alpha_{3}\left(\frac{\alpha_{2}}{\alpha_{3}}-\frac{1,32}{\alpha_{3}}\right)}}=\frac{\alpha_{1}}{1+e^{1,32}}, \\
x\left(t_{C}\right) \approx \frac{\alpha_{1}}{1+e^{-1,32}},
\end{gathered}
$$

also do not depend on $\alpha_{2}, \alpha_{3}$.

Note that point $A$ will be in the right half-plane if and only if $\alpha_{2}>\ln (2+\sqrt{3})$.

From the above it follows that the logistic curve passes through the points $D(0$; $\frac{\alpha_{1}}{1+e^{\alpha_{2}}}$

$B\left(\frac{\alpha_{2}}{\alpha_{3}} ; \frac{\alpha_{1}}{2}\right), C\left(\frac{\alpha_{2}+1,32}{\alpha_{3}} ; 0,79 \alpha_{1}\right)$ (and through point $A\left(\frac{\alpha_{2}-1,32}{\alpha_{3}} ; 0,21\right.$ 
$\left.\alpha_{1}\right)$ at $\alpha_{2}>\ln (2+\sqrt{3})$ ).

\section{Conclusion}

The proposed mathematical methods and models allow to effectively solve the problem of plasticization and modification of high-viscosity polymers, as well as to carry out multicriteria synthesis of composite materials with strictly specified parameters of structure and properties using the quality functionals of kinetic (transient) processes. Practical testing of the proposed method in the synthesis of some polymer composite materials gavepositive results [9-12].

\section{References}

1. Elshin I M, Moschansky N A, Olekhnovich V A, Berman G M 1969 Synthetic resins in construction (Kiev: Publishing house "High school")

2. Solomatov V I 1980 Proceedings of universities. Building and architecture Novosibirsk: P.h. Sibstrin 661

3. Bormotov A N, Proshin I A, Vasilkov A V 2011 Herald of Bryansk Technology University 2(30) 62

4. Bormotov A N, Proshin I A 2009 Herald of Tambov State Technical University 15(4) 916

5. Bormotov A N, Proshin I A, Vasilkov A V 2011 Izhevsk State Technical University Bulletin 2198

6. Bormotov A N, Proshin I A, Vasilkov A V 2011 Izhevsk State Technical University Bulletin 4126

7. Proshin A P, Korolev E V, Bormotov A N, Figovsky O L 2005 Proceedings of the International Conference on Role of Concrete in Nuclear Facilities 2005 International Congress - Global Construction: Ultimate Concrete Opportunities. Ser. "Role of Concrete in Nuclear Facilities - Proceedings of the International Conference" 69

8. Proshin A P, Korolev, E V Bormotov A N 2011 Mathematical modeling and multicriteria synthesis of composite materals (Penza: PSTU)

9. Bormotov A N 2011 Mathematical modeling and multicriteria synthesis of composite materals for special purposes thesis...Doctor of Technical Sciences (Penza: PSTU) $p$ 316

10. Bormotov A N, Proshin I A, Vasilkov A V 2011 Bulletin of Tambov State Technical University 17(2) 542

11. Bormotov A N 2012 Polymer Composite Materials for Radiation Protection (Moscow: P.h. Paleotype) p 272

12. Bormotov A N, Bormotova A A 2018 Regional architecture and construction 3(36) 97 